\title{
PERCEPCIÓN DE LOS QUITEÑOS FRENTE A LÍNEAS DE ESPERA. ESTUDIOS DE ADMINISTRACIÓN
}

\author{
PERCEPTION OF THE PEOPLE FROM QUITO IN FRONT OF WAITING LINES. ADMINISTRATION STUDIES
}

Luis Alberto Dávila Toro ${ }^{1}$ Jaime Gustavo Lastra Vélez ${ }^{2}$

\section{Resumen}

Este artículo está orientado a conocer las preferencias y percepciones de los sistemas de cola de espera de los ciudadanos de Quito. La espera no es una experiencia agradable, y para disminuir sus efectos, es necesario incurrir en gastos que a menudo las empresas no están en capacidad de asumir. Al respecto, muchos autores creen que es posible disminuir la percepción de espera sin incurrir en mayores gastos. Conocer las percepciones de los quiteños al respecto, permitiría aplicar con mayor lógica cualquier tipo de modelo tendiente a mejorar el desempeño del modelo de filas de espera en una organización. Para el efecto se realizó una encuesta sobre la percepción de espera a los ciudadanos de Quito, estudiando aspectos como la apreciación de la espera o tipos de espera máximos.

\section{Palabras clave}

Percepción, teoría de colas, organizaciones, quiteños, modelos.

\section{Abstract}

This article is oriented to know the preferences and perceptions in queuing systems of the citizens of Quito. Waiting is not a pleasant experience, and to diminish its effects, it is necessary to incur expenses that companies are often unable to assume. In this regard, many authors believe that it is possible to decrease the perception of waiting without incurring higher expenses. Knowing the perceptions of the Quito people in this regard, would allow to apply with greater logic any type of model tending to improve the performance of the model of waiting lines in an organization. For this purpose, a survey was conducted on the perception of waiting for the citizens of Quito, studying aspects such as the appreciation of waiting or maximum waiting rates.

\section{Keywords}

Perception, queuing theory, organizations, citizen of Quito, models.

\footnotetext{
${ }^{1}$ Universidad Central del Ecuador. Correo electrónico: luisa.davila@ute.edu.ec.

2 Universidad Tecnológica Equinoccial. Correo electrónico: jaime.lastra@ute.edu.ec.
} 


\section{Introducción}

Para Render y Heizer (1996), la teoría de colas es una parte importante de la administración de operaciones. Las líneas de espera son una situación común en la vida cotidiana, tanto en el sector público como privado, que suelen involucrar transacciones tanto de bienes como de servicios (bancos, restaurantes de comida rápida, etc.) Las características de un sistema de líneas de espera son: las llegadas, la disciplina de la cola y la instalación del servicio.

Al respecto Krajewski y Ritzman (2002) indican que «una línea de espera es cuando uno o más clientes esperan por un servicio» (p.328). Los clientes pueden ser personas u objetos inanimados tales como máquinas que requieren mantenimiento, órdenes de venta aguardando a ser transportadas o artículos del inventario esperando a ser usadas. Una línea de espera se forma a causa de un desbalance temporal entre la demanda por el servicio y la capacidad del sistema para proveer el servicio.

Schroeder (1992) indica que «la situación de las líneas de espera también se denominan problemas de teoría de colas, esto se debe a la aceptación del término británico

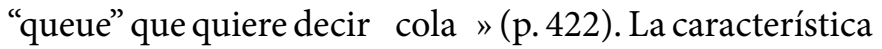
común de estos ejemplos aparentemente diversos es que existe un número de entidades físicas (las llegadas) que buscan recibir servicio de instalaciones limitadas (los servidores). "Como consecuencia, en ocasiones las llegadas deben esperar en una línea su turno de servicio». Muchas veces al llegar a un comercio muchas cajas se encuentran vacías inclusive en días de tráfico o demanda alta, hay que resaltar que el costo de un operador es fijo dado que la legislación actual en el Ecuador no permite la contratación por horas.

Render, Stair, y Hanna (2003) indican que la teoría de colas tiene sus inicios en el trabajo de un danés llamado A.K. Erlang, quien en 1909, experimentó con la demanda en el tráfico telefónico. Ocho años después publicó su investigación y al final de la segunda guerra mundial, su trabajo se extendió de problemas generales a aplicaciones de negocios más específicas. Sin embargo, en el Ecuador, análisis de este tipo son limitados y en muchos casos son privados y no son publicados.

Servicios de calidad demandan calidad en los servicios, entendiéndose que el tiempo que un cliente pasa frente a una cola de espera es tiempo que le resta a su ocio y descanso, tiempo que se le resta al fortalecimiento de su unión familiar, a desarrollarse como persona o a su trabajo, etc.

Desde el punto de vista empresarial, Hayes y Dredge (1998) señanan que el servicio al cliente es sin duda un problema clave para los proveedores de negocios donde las buenas relaciones con los clientes pueden durar muchos años e implican cantidades significativas de dinero. El que un cliente compre en un negocio depende de factores como la confiabilidad, las garantías, la puntualidad, la flexibilidad de la entrega, la relación personal y la facilidad para realizar el pedido o pagar un determinado servicio.

Por otra parte, Robert (2014) manifiesta que uno de los aspectos que permite medir la calidad del servicio es el tiempo de espera de los clientes en un sistema. Al respecto, podemos decir que la teoría de colas es una rama de la investigación operativa cuyo objetivo es tratar de disminuir los costos producto de la formación de la cola de espera, las mismas que según Cao (2002) forman parte de la vida común de muchos de nosotros. Gibson (2016) afirma que las empresas que mejoraron la satisfacción de sus clientes, en el tiempo lograron duplicar su valor accionario, mientras que las que tenían calificaciones de servicio bajas perdieron más del $25 \%$ de su valor.

«El tiempo de espera necesario para atender a tus clientes es uno de los elementos que más influyen en su experiencia de compra, hasta el punto de que puede echar por tierra todos tus esfuerzos en la oferta de un producto o servicio de calidad contrastada y el establecimiento de unos procedimientos de atención al cliente correctos y adecuados a tu público». (Cashlogy, 2016, parr. 5). Las colas de espera generan malestar, ineficiencia, retraso y otros problemas, lo que origina un coste de económico y de tiempo, según Pazos, et. al. (2003) las colas se generan por un desequilibrio temporal entre la demanda del servicio (tasa de arribo) y la capacidad del sistema para suministrarlo. En la teoría de colas clásica, Artalejo (2002) indica que habitualmente, se supone que los usuarios que no consiguen servicio inmediato tras su petición, abandonan el sistema sin intentarlo de nuevo (sistema de perdidas) o permanecen en cola de espera hasta que son atendidos.

De acuerdo con Cao (2002) los modelos de colas de espera están presentes en todas partes, en su banco cuando una persona va a depositar o cobrar su cheque, en el terminal 
terrestre cuando se dirige a tomar el bus que lo lleve a su destino, los aviones tienen que esperar su turno para poder aterrizar, si se pretende renovar la cedula de identidad tendrá que hacer una cola; en resumen un modelo de cola de espera es aquel que tiene una secuencia de elementos (tales como las personas) que llegan a una instalación en busca de servicio, como se muestra en el gráfico siguiente:

Figura 1. Estructura básica de un modelo de colas

$\begin{gathered}\text { Fuente de } \\ \text { entrada }\end{gathered}>\quad$ Cola $>\begin{gathered}\text { Mecanismo } \\ \text { de servicio }\end{gathered}>\begin{aligned} & \text { Clientes } \\ & \text { servidos }\end{aligned}$

Fuente: adaptado de Eppen et al. (2000).

Los objetivos de un modelo de colas de espera son:

- Identificar el nivel óptimo de capacidad del sistema que minimice el costo global del mismo.

- Evaluar el impacto que las posibles alternativas de modificación de la capacidad del sistema tendrían sobre el costo total del mismo.

- Establecer un balance equilibrado entre las consideraciones cuantitativas de costos y las cualitativas del servicio.

- Prestar atención al tiempo de permanencia en el sistema o en la cola de espera.

El objeto del presente estudio es acercarse a la perspectiva de los habitantes de una ciudad media sobre las filas de espera y su percepción sobre las mismas. Dicha ciudad media es Quito, que a 2017 bordea los 2.600 .000 habitantes, con características sociológicas que la diferencian de poblaciones de otras urbes.

\section{Método}

El tema es susceptible de análisis a partir de un modelo. Desde la psicología de la espera, es posible establecer los factores que aumenten o disminuyan la percepción de espera $^{3}$. Pocas cosas son más frustrantes para un cliente como las colas de espera. Sin embargo, Bitran, Ferrer, y Rocha (2008), indican que, con una adecuada gestión de la percepción, una empresa puede reducir la valoración negativa de la espera e incrementar la satisfacción del cliente.

De igual manera Bronchal (2013), indica que desde el punto de vista del cliente, es un tema particularmente sensible la percepción de la calidad en la atención. El tener un enfoque clientelar, es decir, tratar de disminuir el tiempo de espera real, es clave, aunque genere costos en la gestión. Mejorar la percepción de espera es un factor fundamental que afecta la forma en que el cliente valora retrospectivamente la experiencia. Así, una buena gestión de los tiempos en cada paso del proceso puede mejorar la experiencia global del cliente y reportar una ventaja competitiva a empresas de muchos sectores.

Respecto del tiempo de espera y percepción del cliente, se puede afirmar que siempre que exista más de un usuario de un recurso limitado, se puede formar una cola o línea de espera. De la Fuente y Pino Diez (2001), señalan que cuando la cola se compone de objetos inanimados (como materiales, componentes, impulsos eléctricos) que esperan algún tipo de procesamiento (por ejemplo una maquina), el problema es básicamente económico: ¿qué longitud debe tener la cola para minimizar los costos de mantener un inventario y/o aumentar una máquina adicional? Cuando la cola está formada por personas que esperan un servicio, el problema tiene muchos aspectos psicológicos además de los económicos, los mismos que a menudo son muy difíciles de cuantificar.

Los clientes recuerdan los aspectos más destacados de la experiencia. Así, probablemente no tendrán en cuenta la duración del servicio si se trata de un factor secundario. Por ejemplo, los clientes a quienes se entretiene con música o programas de televisión probablemente olvidarán el tiempo que se les ha hecho esperar. En este caso, según Bitran, Ferrer, \& Rocha (2008), la duración del servicio, aunque sea larga, acaba siendo un factor neutral y no negativo.

En todo caso la percepción de espera del cliente podría ser mayor o menor de lo que realmente es, Bronchal (2013) señala que dependiendo de factores como tiempo disponible, clima, facilidades de parqueo etc. Si el tiempo de espera es corto pero el entorno genera impaciencia o ansiedad, los clientes podrían tener la sensación de que han esperado demasiado. Si, por el contrario, se los hace esperar mucho, pero el entorno es el apropiado, les parecerá que el tiempo ha sido relativamente corto.

El Instituto Nacional de Estadísticas y Censos (2017) indica que en el Distrito Metropolitano de Quito existen

3 Se entiende como la percepción la función psíquica que permite al organismo, a través de los sentidos, recibir, elaborar e interpretar la información proveniente de su entorno. 
con corte a fines del año 2017 un total de 1'911,966 personas, de las cuales el $51.70 \%$ son mujeres y el restante $48.30 \%$ son hombres. Es así como para el cálculo de nuestra muestra se trabajó con una varianza desconocida (0.50 x 0.50), un $4.68 \%$ de error y un coeficiente de confianza del 95.5\% (2 desviaciones respecto de la media poblacional); dando como resultado una muestra de 456 personas de ambos sexos tal como se muestra en la Tabla 1.

Tabla 1. Sexo de los encuestados.

\begin{tabular}{|l|c|c|c|c|c|}
\hline \multicolumn{1}{|c|}{ Sexo } & Población & Muestra & Porcentaje & $\begin{array}{c}\text { Porcentaje } \\
\text { valido }\end{array}$ & $\begin{array}{c}\text { Porcentaje } \\
\text { acumulado }\end{array}$ \\
\hline Femenino & 988,486 & 237 & 52.0 & 52.0 & 52.0 \\
\hline $\begin{array}{l}\text { Mascu- } \\
\text { lino }\end{array}$ & 923,480 & 219 & 48,0 & 48,0 & 100,0 \\
\hline Total & $1,911,966$ & 456 & 100 & 100 & \\
\hline
\end{tabular}

Fuente: elaboración propia

\section{Resultados}

A fin de determinar los tiempos tolerables de espera de los quiteños se realizó una investigación de campo en noviembre de 2017, a fin de determinar: ¿qué piensan los ciudadanos de la capital al respecto? La encuesta se realizó en diferentes sectores de la ciudad de Quito, como se aprecia en la figura 2.

Figura 2. Sector donde viven encuestados

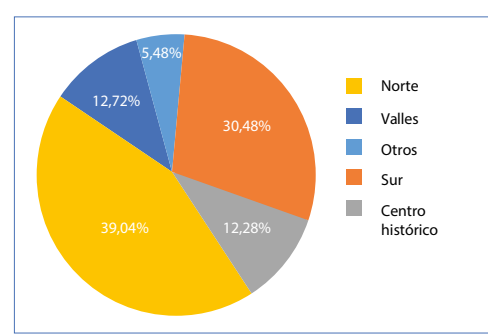

Fuente: elaboración propia.

Se preguntó a los encuestados: ¿cuál es tiempo tolerable de espera por rangos de tiempo en una cola?, las respuestas fueron las siguientes:

Figura 3. Tiempo de espera tolerable

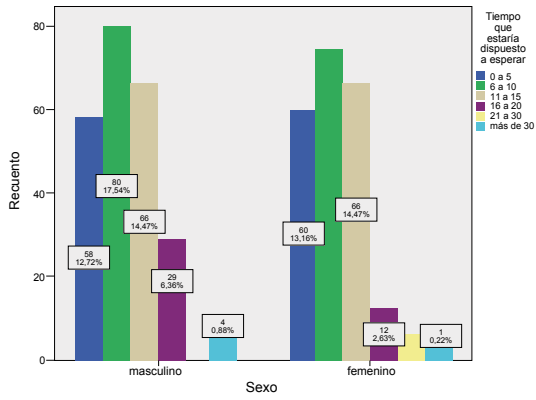

Calculando el tiempo de espera promedio de los quiteños se observa que fue de 9 minutos con 5 segundos. Cuando un cliente espera un sistema más allá de este tiempo de espera tolerable se considera que el sistema está saturado. Esto quiere decir que los servidores se encuentran al tope de su capacidad, entendiéndose como personas que atienden en un determinado servicio. Al realizar el cruce del tiempo de espera por sexo de los encuestados se observan los siguientes datos, según lo explica la Tabla 2:

Tabla 2. Tiempo de espera tolerable por sexo.

\begin{tabular}{|l|c|c|c|c|c|c|c|}
\hline $\begin{array}{c}\text { Tiempo } \\
\text { de espera }\end{array}$ & $\begin{array}{c}\mathbf{0} \text { a } \mathbf{5} \\
\text { Min. }\end{array}$ & $\begin{array}{c}\mathbf{6} \text { a } \mathbf{1 0} \\
\mathbf{M i n} .\end{array}$ & $\begin{array}{c}\mathbf{1 1} \text { a } \mathbf{1 5} \\
\mathbf{M i n} .\end{array}$ & $\begin{array}{c}\mathbf{1 6} \text { a } \mathbf{2 0} \\
\mathbf{M i n}\end{array}$ & $\begin{array}{c}\mathbf{2 1} \text { a } \mathbf{3 0} \\
\mathbf{M i n}\end{array}$ & $\begin{array}{c}\text { mas de } \\
\mathbf{3 0} \text { Min. }\end{array}$ & Total \\
\hline Masculino & 58 & 80 & 66 & 29 & 0 & 4 & 237 \\
\hline Femenino & 60 & 74 & 66 & 12 & 6 & 1 & 219 \\
\hline total & 118 & 154 & 132 & 41 & 6 & 5 & 456 \\
\hline
\end{tabular}

Fuente: elaboración propia

Se observa que la predisposición de espera de las mujeres es menor que la de los hombres, así los hombres tienen una predisposición de espera promedio de 9 minutos con 21 segundos, mientras que las mujeres tienen una predisposición de espera promedio de 8 minutos 47 segundos.

Al realizar la prueba de hipótesis del chi cuadrado, la hipótesis de investigación se acepta en virtud de que dicho valor es mayor a 2.40 , es decir, existen diferencias de percepción de espera entre hombres y mujeres. Cabe indicar que existen en la Tabla 2 un número de seis categorías, razón por la cual los grados de libertad son de $\mathrm{k}$ - 1 que da como resultado 5. El p-value o significación asintótica es menor a $0.025(0.05 / 2)$, razón por la cual se rechaza la hipótesis de independencia $\mathrm{H}_{0}$ de no relación entre las variables (según la Regla de decisión de Fisher), se acepta la hipótesis alternativa $H_{1}$, que indica que la relación entre las variables existe y es estadísticamente significativa y no se debe al azar.

Tabla 3. Pruebas de chi-cuadrado

\begin{tabular}{|l|c|c|c|}
\multicolumn{1}{|c|}{ Cálculo } & Valor & Grados de Libertad & Sig. asintótica (bilateral) \\
\hline $\begin{array}{l}\text { Chi-cuadrado } \\
\text { de Pearson }\end{array}$ & $14.428^{4}$ & 5 & 0.013 \\
\hline $\begin{array}{l}\text { Razón de } \\
\text { verosimilitudes }\end{array}$ & 17.068 & 5 & 0.004 \\
\hline $\begin{array}{l}\text { No. de casos } \\
\text { válidos }\end{array}$ & 456 & & \\
\hline
\end{tabular}

Fuente: elaboración propia

En igual sentido se ha realizado un cruce de tiempo de espera de acuerdo con la ocupación de las personas, a continuación, se muestra la siguiente información.

Fuente: elaboración propia 
Figura 4. Tiempo de espera tolerable por ocupación.

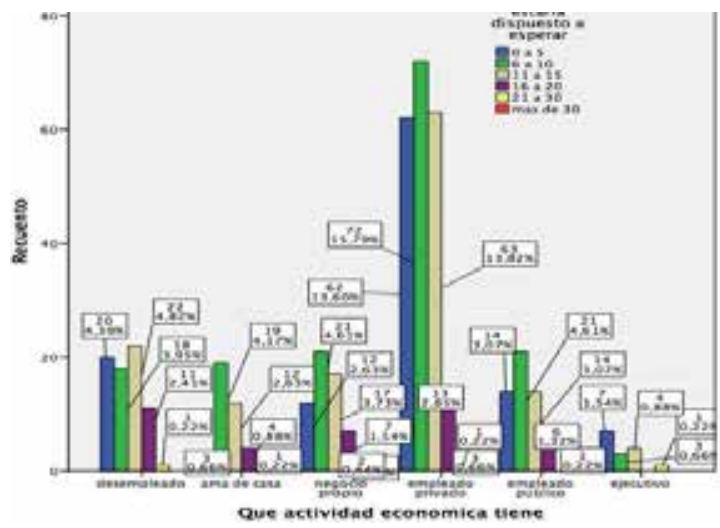

Fuente: elaboración propia

Al realizar la prueba de hipótesis del chi cuadrado la hipótesis de investigación se acepta en virtud de que dicho valor es mayor a 0.05 , es decir no existe diferencia en la percepción de espera de acuerdo con las actividades económicas de los encuestados.

Tabla 4. Pruebas de chi-cuadrado Actividad Económica vs. Tiempo de espera.

\begin{tabular}{|l|c|c|c|}
\multicolumn{1}{|c|}{ Cálculo } & Valor & $\begin{array}{c}\text { Grados de } \\
\text { libertad }\end{array}$ & $\begin{array}{c}\text { Sig. asintótica } \\
\text { (bilateral) }\end{array}$ \\
\hline Chi-cuadrado de Perason & $31.730^{5}$ & 25 & 0.166 \\
\hline Razón de verosimilitudes & 34.351 & 25 & 0.101 \\
\hline$N^{\circ}$ de casos válidos & 456 & & \\
\hline
\end{tabular}

Fuente: elaboración propia.

Figura 5. Tiempo de espera tolerable por sector que habitan.

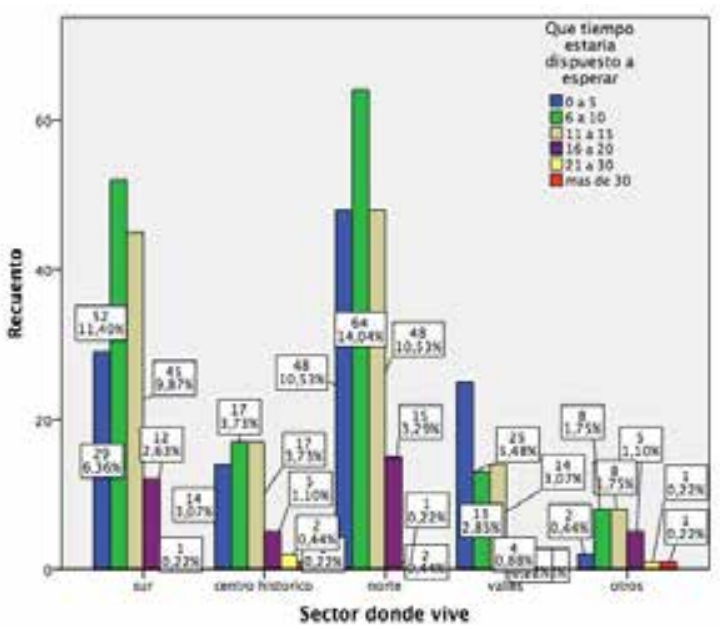

Fuente: elaboración propia
Al igual que los otros casos se aplicó una prueba chicuadrado para saber la validez de los datos presentados.

Tabla 5. Pruebas Chi cuadrado Tiempo de espera vs. Sector

\begin{tabular}{|l|c|c|c|}
\multicolumn{1}{|c|}{ Cálculo } & Valor & $\begin{array}{c}\text { Grados de } \\
\text { libertad }\end{array}$ & $\begin{array}{c}\text { Sig. asintótica } \\
\text { (bilateral) }\end{array}$ \\
\hline Chi-cuadrado de Perason & $31.730^{5}$ & 25 & 0.166 \\
\hline Razón de verosimilitudes & 34.351 & 25 & 0.101 \\
\hline$N^{\circ}$ de casos válidos & 456 & & \\
\hline
\end{tabular}

Fuente: elaboración propia

De las Tablas 4 y 5 se puede concluir que el p-value o significación asintótica es mayor a $0.025(0.05 / 2)$ con valores de 0.166 y 0.105 respectivamente, razón por la cual se acepta la hipótesis de independencia H0 de no relación entre las variables-según la regla de decisión de Fisher-, se rechaza la hipótesis alternativa $\mathrm{H} 1$, que indica que la relación entre las variables existe y es estadísticamente significativa y no se debe al azar. Lo cual significa que no existe relación entre el sector en que viven los encuestados o su actividad económica con el tiempo que están dispuestos a esperar. También se preguntó: ¿dónde usted considera que las colas son más largas en el sector privado o en el sector público?, obteniéndose los siguientes resultados.

Figura 6. ¿Dónde considera usted que las filas de espera son más largas? ${ }^{7}$

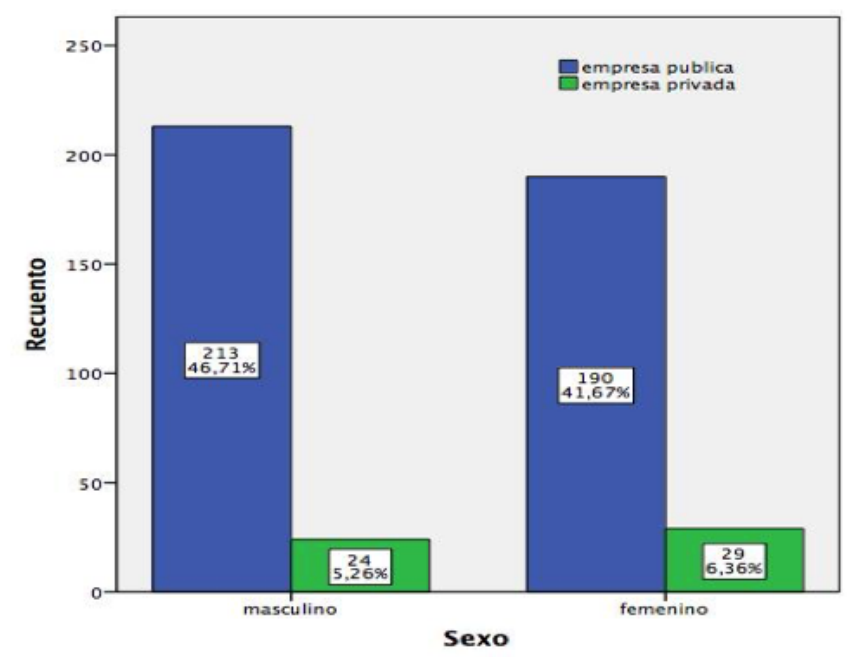

Fuente: elaboración propia

${ }^{4} 4$ casillas (33.3\%) tienen una frecuencia esperada inferior a 5. La frecuencia mínima esperada es 2.40

${ }^{5} 16$ casillas $(44.4 \%)$ tienen una frecuencia esperada inferior a 5 . La frecuencia mínima esperada es 0.16

${ }^{6} 16$ casillas $(36.7 \%)$ tienen una frecuencia esperada inferior a 5. La frecuencia mínima esperada es 0.27

${ }^{7}$ Escala Ordinal (5 más importante -1 menos importante). 
Se consultó los factores que a juicio de los encuestados disminuyen la percepción de espera, para el efecto se aplicó una escala ordinal y se procesaron los datos de acuerdo con la ponderación de los factores multiplicado por el valor máximo obteniéndose los siguientes resultados:

Figura 7. ¿Qué factores a su juicio ayudan a disminuir la percepción de espera?

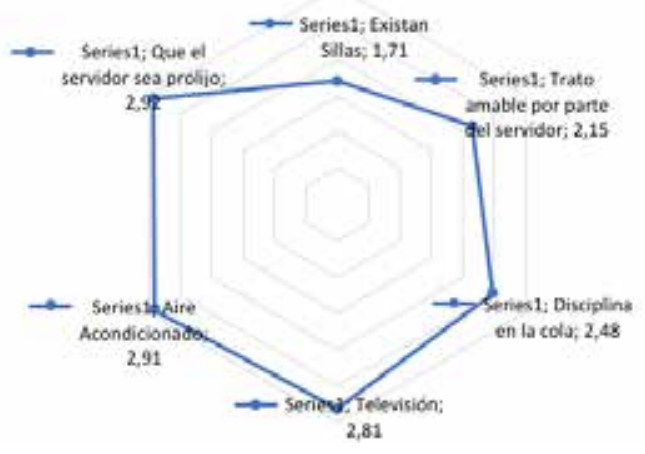

Fuente: elaboración propia

El factor que más relevancia tiene por parte de los encuestados es que el servidor sea prolijo en su trabajo, seguido del aire acondicionado en días de calor o el uso de medios visuales que permitan esperar de una manera en que la percepción del tiempo no sea tan notoria. Es decir, a los clientes les enfada sobre manera que los dependientes no cumplan con su obligación de atender a los clientes. También en caso de que se generen colas en lugares cerrados o con mucho frío sería importante contar con un sistema adecuado que disminuya los efectos que se producen por el clima y que pueden incidir en el ánimo de los usuarios. Finalmente, un sistema de audio y video podría mejorar el ánimo de los clientes el momento de la generación de líneas de espera.

\section{Discusión y conclusiones}

Existe evidencia de que el tiempo de espera en un sistema podría afectar la satisfacción clientelar y redundar en una disminución de sus clientes. Cuando un cliente espera un tiempo mayor a diez minutos se considera que el sistema se encuentra saturado. Independientemente del sector donde resida o de la actividad económica, la gente percibe que pierde tiempo al no ser atendido de una manera rápida por el sistema.

Para disminuir el tiempo de espera existen varias formas, siendo la más recomendable el aumento de servidores. El estudio indica que existe una menor predisposición de espera por parte de los hombres frente a las mujeres; de 6 a 10 minutos un $17.54 \%$ frente a un $16.23 \%$ respectivamente. El aumento de servidores ocasiona a la empresa un incremento de gastos que debe ser comparado con la disminución de los costos de espera del cliente para equilibrar el modelo. Las personas encuestadas pertenecen a la población económicamente activa con un promedio de edad de 32 años, una mediana de 29 y una moda de 22 y una desviación estándar de 10 años.

Independientemente de su actividad, el 88\% las personas están dispuestas a esperar hasta quince minutos, a partir de ese momento la satisfacción disminuye con un menor porcentaje, esto se corrobora con la prueba de hipótesis que originó como resultados valores $\mathrm{p}$ (p-value) altos, aceptando H0. Cuando el cliente supera el límite de la paciencia existe una alta probabilidad de que abandone el sistema.

El tiempo de espera en un sistema podría afectar la satisfacción clientelar y redundar en una disminución de sus clientes. El coeficiente de verosimilitud y el chicuadrado son similares, en cualquiera de los cálculos realizados, dado que la muestra es un reflejo de la población.

Un sistema de colas de espera bien gestionado no debe representar para una empresa un costo, porque se podría compensar con un aumento de ingresos. Las empresas también pueden mejorar la percepción de espera del cliente. A menudo esta percepción es menos costosa que el aumento de servidores. Deben crearse veedurías ciudadanas o comités que generen una presión en la mejora en el servicio.

La forma más recomendable de disminuir la percepción de espera es tener servidores atentos y eficientes. Se proponen a futuro análisis por industrias dado que según Render y Heizer (1996), existen varios modelos de líneas de espera: llegadas que siguen una distribución de Poisson; llegadas que siguen una disciplina FIFO (PEPS) y llegadas con una fase de servicio única. Estos pueden variar dependiendo el tipo de industria inclusive dependiendo el tipo de negocio.

Katz, Larson, y Larson (1991) sugieren que para mejorar un sistema que no abastece a sus usuarios, se determine un tiempo de espera aceptable para los clientes. También se recomienda intentar desviar la atención de los clientes mientras esperan y mejorar las condiciones ambientales. Moderadores ambientales, bien elegidos, entretienen 
a los clientes mientras esperan. Una buena decisión es informar a los usuarios que deben esperar, reducir la incertidumbre de la espera aumenta la sensación de control de los clientes y, por lo tanto, su grado de satisfacción.

Los mismos autores recomiendan que se mantenga a los empleados que no están atendiendo clientes fuera de la vista de estos. Se debe segmentar a los clientes y enseñar a los servidores a ser amables, también se puede educar o sugerir a los clientes a venir durante periodos de menos congestión. En resumen, se debe tener una perspectiva de largo plazo para deshacerse de las filas.

Independientemente de la cultura, la teoría de colas ha ayudado a resolver problemas que en un mundo globalizado necesitan soluciones rápidas. Actualmente con la inteligencia artificial y los datos que generan los vehículos, carreteras o semáforos se pueden planificar nuevas inversiones o predecir futuras congestiones que necesitan ser resueltas en función del tráfico de las ciudades. En EE.UU. el departamento de transporte ha aprobado la transferencia de datos que se generan de la temperatura de los vehículos a la red pública para controlar justamente líneas de espera en carreteras y ciudades.

\section{Referencias}

1. Artalejo, F. (2002). Standard and retrial queueing system: A Comparative analysis. Revista Matemática Complutense 15(1), 101-129.

2. Cashlogy (2016). ¿Por qué es importante reducir el tiempo de espera de nuestros clientes? Recuperado de: http://cashlogy.es/reducir-tiempo-esperaclientes/

3. Bitran, G., Ferrer, J. C., y Rocha, P. (2008). Om Forum - Managing Customer Experiences: Perspectives on the Temporal Aspects of Service Encounters. Manufacturing \& Service Operation Management, 10(1), 61-83.

4. Bronchal, J. M. (2013). Gestion de Colas de Espera: Cómo mejorar la percepción de mis clientes en las esperas. Obtenido de: http://www.projectlinkr.com/ blog/gesti-n-de-colas-de-espera-c-mo-mejorar-lapercepci-n-de-mis-clientes-en-las-esperas/

5. Cao Abad, R. (2002). Introducción a la Simulación y

\section{a la Teoría de Colas. A Coruña: NETBIBLO.}

6. De la Fuente, D., y Pino Diez, R. (2001). Teoría de Líneas de Espera - Modelos de Colas. Oviedo: Universidad de Oviedo Servicio de Publicaciones.

7. Eppen, G. D.; Weatherford, Larry R.; Schmidt, C. P.; Moore, Jeffrey H.; Gould, F. J. (2000). Investigación de Operaciones en la Ciencia Administrativa. México: Prentice Hall.

8. Gibson, P. (2016). The World of Customer Service. Arizona: South -Western CENGAGE - Learning.

9. Hayes, J., y Dredge, F. (1998). Managing Customer Service. London: Gower Publishing Limited.

10. Instituto Nacional de Estadísticas y Censos (2017). Ecuador en Cifras. Obtenido de: http://www. ecuadorencifras.gob.ec/tras-las-cifras-de-quito/

11. Katz, K. L., Larson, R. C., y Larson, B. M. (1991). Prescription for Waiting-in Line Blues: Entertain, Enlighten and Engage. Sloan Management review,(winter), 32(2), 44-53.

12. Krajewski, L. J., y Ritzman, L. P. (2002). Operations Management Strategy and Analysis (Sixth Edition ed.). New Jersey, United States: Pearson.

13. Pazos, J. J., Suárez, A., y Díaz, R. (2003). Teoría de Colas y simulación de envetos discretos. Madrid: Pearson.

14. Render, B., y Heizer, J. (1996). Principios de Administración de Operaciones. México: Pearson.

15. Render, B., Stair Jr., R. M., y Hanna, M. E. (2003). Quantitative Analysis for Management. New Jersey: Pearson.

16. Robert, L. (2014). Customer Service Skills for Success. New York: McGraw-Hill Higher Education.

17. Schroeder, R. G. (1992). Administración de Operaciones Toma de decisiones en la función de operaciones. México: McGraw-Hill. 